\section{ANÁLISIS DE ESCORIAS FÉRREAS: NUEVAS APORTACIONES AL CONOCIMIENTO DE LA SIDERURGIA PRERROMANA EN ESPAÑ̃ (1)}

\author{
IRON SLAGS ANALYSIS: NEW \\ CONTRIBUTIONS TO THE \\ KNOWLEDGE OF PRE-ROMAN IRON \\ INDUSTRY IN SPAIN
}

PABLO GÓMEZ RAMOS (*)

\begin{abstract}
Iron Age in Spain started with the arrival of the Phoenicians, and it meant the beginning and spread of iron production. Though many archaeological references exist, reliable information about the technology to produce the new metal is scarce. In this article, an analytical and microscopical study of smelting slags and furnace linings is presented reaching the conclusion that pre-Roman iron production was based on small size furnaces, usually without tapping slags. The slags, of irregular compositions, were of fayalite type, containing large amounts of wüstite, magnetite and free silica on some occasions. All these features indicate an unsophisticated iron smelting technology during this period.
\end{abstract}

Palabras clave: Análisis. Edad del Hierro. Escorias. Hornos de fundición. Siderurgia.

Key words: Analysis. Iron Age. Slags. Smelting furnaces. Iron production.

\section{INTRODUCCIÓN}

El origen de la metalurgia del hierro en la Península Ibérica ha sido tema de diversos es- 
tudios y enconados debates. La idea tradicional que atribuía la introducción del hierro a invasores centroeuropeos llegados de allende los Pirineos, tiempo ha que fue desechada para relacionarla con la presencia de comerciantes o colonos semitas en el Mediterráneo Occidental. Así, la producción más antigua de hierro está constatada en los enclaves fenicios de la costa malagueña, como ejemplifica el hallazgo de escorias de hierro, restos de hornos y otros elementos relacionados con la fundición - también de cobre - en El Morro de Mezquitilla (Málaga) fechado a fines del siglo IX a.C. y principios del VIII, y en el Peñón de Toscanos y Cerro del Mar, en el siglo VII a.C. A estos hallazgos hay que unir el descubrimiento de piezas aisladas de hierro en contextos del Bronce Final, en un número cada vez mayor de yacimientos y que ha servido para replantear la hipótesis de contactos precoloniales con anterioridad al establecimiento de los primeros enclaves fenicios (Almagro-Gorbea, 1993).

Sin embargo, frente a la relativa abundancia de datos arqueológicos sobre la introducción del nuevo metal en la Península Ibérica o la aparición más temprana de los primeros objetos férreos en ambientes de finales de la Edad del Bronce, escasean los estudios tecnológicos. En efecto, se desconocen casi totalmente aspectos relacionados con la reducción de minerales en hornos, minería, producción de materia prima en lingotes, tratamientos posteriores del metal, etc. Los únicos estudios metalúrgicos y/o metalográficos, como veremos más adelante, proceden de unos pocos poblados de la $\mathrm{Me}$ seta Norte o de artículos puntuales dedicados al estudio de los residuos metalúrgicos en varios poblados de la Segunda Edad del Hierro en la región de Aragón.

Es en este contexto donde integramos nuestro trabajo. Apoyándonos en el análisis empírico de numerosas piezas relacionadas con la fundición primaria de hierro trataremos de demostrar que la siderurgia prerromana, al menos en España, se basó en una tecnología de hornos sencillos, con baja productividad, y por tanto, similar a la del trabajo del cobre y del bronce (Gómez Ramos, 1996a). No obstante, ambas metalurgias presentan diferencias importantes derivadas del proceso mismo de la fundición, por lo que resulta procedente comenzar presentando algunos datos referidos al funcionamiento general de los hornos de hierro. Queremos señalar que se ha dejado de lado el tema del trabajo del hierro posterior a su obtención, ya que su estudio no aporta datos significativos respecto a los hornos y minerales utilizados en la producción primera de hierro.

\section{LA REDUCCIÓN DE LOS MINERALES DE HIERRO}

La siderurgia antigua benefició para la obtención de hierro los abundantes depósitos minerales de óxidos, hidróxidos y carbonatos de hierro (hematites, limonita, magnetita, etc.). La reducción de estos minerales se hacía con carbón vegetal en los hornos de fundición. Sin embargo, a diferencia de lo que ocurre con otros metales, los hornos siderúrgicos presentan como característica principal (al menos hasta época medieval avanzada) la imposibilidad de alcanzar en ellos la temperatura de fusión del hierro puro (aproximadamente $1.560^{\circ} \mathrm{C}$ ) y por lo tanto, su licuado y trabajo en moldes. El producto principal obtenido en el horno era un amasijo sólido de hierro dulce y escoria que podía alcanzar varios kilogramos de peso, denominado con los vocablos esponja ferrífera, lupia, o bloom, término inglés que también sirve para designar estos hornos como bloomery furnaces.

Ello se debe en gran medida al combustible empleado. El carbón vegetal utilizado en la antigüedad no tenía el poder calorífico suficiente para licuar el hierro, cosa que no se conseguió hasta que comenzó a explotarse el carbón mineral ya que con él había la posibilidad de que se formara una aleación hierro-carbono que funde a temperatura más baja. Así, según el diagrama Hierro-Carbono, con un $4,3 \%$ de carbono en disolución (2) se consigue hierro líquido a $1.130^{\circ} \mathrm{C}$. La dificultad estribaba, en conseguir dicha disolución con los hornos primitivos,

(2) El hierro sólido tiene la propiedad de disolver carbono por absorción, a alta temperatura. Esta propiedad es la base de la obtención de aceros carburados en fragua en las herrerías antiguas.

T. P., 53, n. ${ }^{\circ} 2,1996$ 
pues los análisis de esponjas ferríferas de La Tène no dan tasas de carbono superiores al 0'5\% (Tylecote, 1976: 44, tabla 30), y a esa concentración el hierro funde a los inalcanzables $1.560^{\circ} \mathrm{C}$.

Las lupias debían trabajarse posteriormente por martilleado o forja en caliente para eliminar la mayor cantidad posible de escoria y formar el lingote. Lupias y lingotes manufacturados de hierro que son singularmente escasos en los contextos arqueológicos peninsulares.

No es una tarea sencilla tipificar los hornos. En las excavaciones arqueológicas casi nunca aparecen intactos, las interpretaciones son variopintas y la literatura científica es tan extensa que se corre el riesgo de perderse en tipologías subjetivas basadas casi siempre en aspectos meramente formales. Partiendo de una primera clasificación general establecida por Coghlan en 1956 (1977), en la que se establecían hornos en forma de cuenco, hornos abovedados y hornos en forma de chimenea y que fue ampliada o modificada por otros autores como Cleere (1972) o Forbes (1972), actualmente se reconocen dos tipos básicos deducidos de las observaciones etnográficas y de los trabajos experimentales:

Horno de cubeta. Aparece mencionado en la bibliografía última con el término bowl furnace. Puede tener una construcción superior de forma troncocónica o abovedada, pero el factor a tener en cuenta para su diferenciación con el horno vertical de chimenea es que existan medidas similares entre la altura y la anchura (Tylecote y Merkel, 1985).

Horno de chimenea, cuando la altura es netamente superior a la anchura del horno. A lo largo de la operación la escoria más o menos líquida caía al fondo de la solera, excavada en el suelo o no, y al igual que en los hornos de cobre, también era posible sangrarla fuera de la cámara; la diferencia estriba en la posición de las piqueras de sangrado, que en el caso del cobre ha de estar más alta, pues la escoria flota sobre el metal, y en el hierro sucede lo contrario, por lo que la salida ha de estar en el punto más bajo. En ambos casos, se trata siempre de hornos de pequeño diámetro que no suelen superar los $50 \mathrm{~cm}$. con una altura aproximada de un metro, y no deben con- fundirse en ningún caso con los altos hornos capaces de obtener hierro líquido, característicos por sus altas chimeneas que llegaron a alcanzar en Europa Occidental a finales del siglo XIII los $5 \mathrm{~m}$., o los $8 \mathrm{~m}$. en el siglo XVIII y que contaban con potentes sistemas de aireación y carbón mineral como combustible.

Evidentemente, es difícil distinguir a través de los restos arqueológicos un horno de cubeta de un horno de chimenea ya que las partes estructurales superiores generalmente no se han conservado. La división útil o práctica en el terreno arqueológico estribará en si el horno, ya fuera de cubeta o de chimenea, tuvo o no piquera para el sangrado al exterior de sus escorias. Las escorias de sangrado se distinguen por mostrar en sus superficies signos evidentes de haber fluido en estado pastoso y se diferencian fácilmente de las escorias que quedan depositadas en el fondo del horno y que originan características tortas de forma plano-convexa. Ésta es la única división cierta (hornos con o sin sangrado de sus escorias) cuando los restos arqueológicos son realmente parcos, como ya señalara Tylecote (1987: 162).

\section{VESTIGIOS RELACIONADOS CON LA SIDERURGIA PRERROMANA EN ESPAÑA}

Los hallazgos vinculados con tareas de producción de hierro (hornos, escorias y minerales) durante el último milenio a.C. son abundantísimos a juzgar por las referencias escritas (3). Sin embargo, un número elevado de ellos, tanto españoles como portugueses, se refieren simplemente a escorias localizadas en tal o cual yacimiento pero sin presentar ni análisis ni descripciones. También es destacable la ausencia casi total de lupias, lingotes y revestimientos de hornos. La documentación incluye todas las provincias españolas así como nume-

(3) El total de estas referencias abarca 293 yacimientos, que divididos por regiones quedan del siguiente modo: Portugal, 12; Andalucía, 49; Extremadura, 16; Galicia, 31; Asturias y Cantabria, 6; País Vasco, 11; Navarra, 3; Rioja, 1; Aragón, 46; Meseta Norte, 44; Meseta Sur, 13; Cataluña, 31; Comunidad Valenciana, 20; Murcia, 7; e islas Baleares, 3.

$$
\text { T. P., 53, n. }{ }^{\circ} 2,1996
$$


rosas zonas de Portugal, y en su mayor parte se encuadra cronológicamente en un momento avanzado del I milenio a.C. En este trabajo centraremos nuestra atención, por lo tanto, solamente en los mejor documentados y que se encuentran en España, remitiendo para el resto al estudio detallado de Gómez Ramos (1996b).

La información más interesante proviene de los yacimientos fenicios de la costa de Málaga. El Morro de Mezquitilla (Algarrobo) ha proporcionado los restos de fundición fenicios más antiguos de la Península Ibérica, fechables a fines del siglo IX y principios del siglo VIII a.C. (Schubart, 1985a; 1985b: 63 y 1990: 38). En la zona llamada "talleres", correspondiente a los estratos más antiguos del yacimiento, se hallaron varios hornos, escorias y toberas o boquillas de tobera de uno y dos conductos, algunas con restos de metal adheridos. Tanto H. Schubart en sus distintos trabajos como Keesmann y Hellermann (1989: 92-117) abogan por la existencia de un taller de herrero con escorias de forja y no una fundición para obtener el metal. También El Cortijo, El Cerro del Peñón de Toscanos y Cerro del Mar (Torre del Mar) han proporcionado un elenco de materiales relacionados con la producción metalúrgica de cobre y hierro, compuesto por hornos, vasijas-horno, toberas, escorias, minerales, etc., fechado a partir de mediados del siglo VIII a.C. (Schubart y Niemeyer, 1969: 209, Fig. 3; Niemeyer, 1982: 116-118; Schubart y Maass-Lindemann, 1984: 147-148; Keesmann et alii, 1989: 99-108; y Schubart y Arteaga, 1986: 509).

En la provincia de Murcia se encuentra El Castellar de Librilla (Ros Sala, 1989 y 1993: 71109). Su importancia viene motivada por el hallazgo de restos de dos estructuras que han sido clasificadas como hornos metalúrgicos para el procesamiento de minerales de hierro en un horizonte relativamente antiguo, uno de la segunda mitad del siglo VIII-VII a.C. y otro en un momento más avanzado que abarcaría aproximadamente los siglos VI-V a.C. Las descripciones morfológicas y de funcionamiento que presenta la autora son discutibles ya que hablan de minerales contenidos en crisoles en el interior de una supuesta estructura cupular de adobes de grandes dimensiones. No obstante, los análisis de escorias y minerales efectuados por Arana y Pérez Sirvent (1993: 111-129) indican sin duda la producción de hierro en este poblado indígena.

Dentro de la II Edad del Hierro cabe destacar, entre otros, el hallazgo de escorias y minerales en el yacimiento soriano de Castilmontán (Somaén) cuyo estudio analítico fue realizado por Madroñero et alii (1992). También contamos con análisis de una serie de escorias de reducción y de forja procedentes de distintos castros prerromanos de la provincia de Zamora como La Dehesa de Amor (La Tuda) y El Cerco de Sejas (Aliste) (Esparza, 1987: 285-287 y 399). Existen escorias analizadas de los poblados aragoneses de Monte Catma (La Ginebrosa) (Martín Costea et alii, 1993: 241-283), Vallipón (Castellote) (Martín Costea y Ruiz Zapatero, 1980: 31-40), en ambos casos con la mina de hierro cerca del asentamiento, y La Oruña (Veruela), nó lejos del metalotecto ferrífero del Moncayo (Hernández Vera y Murillo, 1985: 181-184). Los pocos estudios analíticos de los restos de fundición inciden en producciones metalúrgicas a pequeña escala con explotación de los recursos minerales locales de cada zona, como ilustran las composiciones de las escorias analizadas.

Todos estos datos se encuadran dentro de un panorama historiográfico en el que predominan ideas equivocadas sobre los hornos de fundición y la metalurgia extractiva del hierro que ya pusimos de relieve en nuestro trabajo doctoral (Gómez Ramos, 1996b). En cuanto a los hornos, porque muchas de las estructuras ideadas no cumplen los requisitos para que pudieran funcionar como tales, y en cuanto a la metalurgia, porque se toma como modelo de la fundición del hierro el del cobre y bronce, cosa que no puede ser así puesto que el hierro dulce o ligeramente acerado de la Prehistoria nunca pudo ser derretido en crisoles y vertido en moldes debido a su elevado punto de fusión, inalcanzable con los medios disponibles entonces, como se ha argumentado anteriormente. Las reconstrucciones y funcionamientos planteados para los hornos de Librilla en Murcia (Ros Sala, 1989 y 1993) o los pacenses de Belén y Los Castillejos 2 (Berrocal, 1993), con la utilización de "crisoles" para efectuar en su interior la fundición de minerales o de hierro metálico, "toberas" para el sangrado de escorias, etc.,

T. P., 53, n. ${ }^{\circ} 2,1996$ 


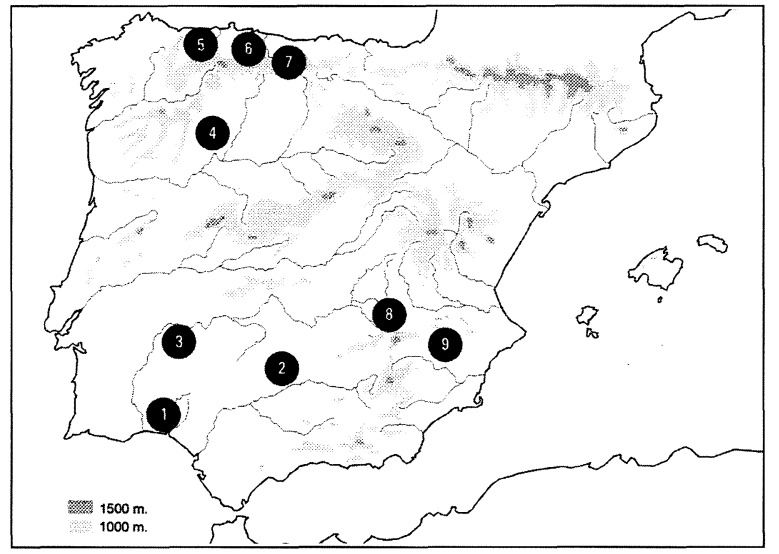

Fig. 1. Distribución de yacimientos con escorias de hierro analizadas. 1. Calle del Puerto 10 (Huelva). 2. Llanete de los Moros (Córdoba). 3. Castrejón de Capote (Badajoz). 4. Corona de Corporales (León). 5. Carcedo (Asturias). 6. Campa Torres (Asturias). 7. El Picu Castiellu (Asturias). 8. Cerro de las Nieves (Ciudad Real). 9. El Amarejo (Albacete).

junto a hornos de varios metros de diámetro como por ejemplo, los hornos de La Oruña en Zaragoza (Hernández Vera y Murillo Ramos, 1985) son sintomáticos a este respecto.

Con la investigación que hemos llevado a cabo sobre numerosos materiales relacionados con la producción de hierro, especialmente escorias (Fig. 1), enviados por arqueólogos para su estudio en el laboratorio mediante espectrometría por fluorescencia de rayos X (energía dispersiva) (4) y microscopia óptica y electrónica en el I.C.R.B.C., Museo de América y Universidad Autónoma de Madrid, se amplía de manera notable el corpus de datos analíticos con los que poder precisar nuevos conocimientos de la siderurgia prerromana en España.

Aunque el estudio de los materiales no nos permite conocer la morfología concreta de ningún horno, hemos analizado un fragmento de revestimiento del yacimiento asturiano de $\mathrm{La}$

(4) En los análisis por fluorescencia de rayos $\mathrm{X}$ se han calculado los valores respectivos de los elementos en cuentas por segundo (análisis espectral), en las siguientes líneas: $\mathrm{Fe}(\mathrm{K} \alpha), \mathrm{Mn}(\mathrm{K} \alpha), \mathrm{Ca}(\mathrm{K} \alpha), \mathrm{Ba}(\mathrm{K} \alpha), \mathrm{Cu}(\mathrm{K} \alpha), \mathrm{As}(\mathrm{K} \beta)$, $\mathrm{Sb}(\mathrm{K} \alpha), \operatorname{Ag}(\mathrm{K} \alpha), \mathrm{Sn}(\mathrm{K} \beta)$ y $\mathrm{Pb}(\mathrm{L} \beta)$. No se han podido determinar los elementos más ligeros que el calcio debido a la configuración del espectrómetro. Estos análisis por fluorescencia han sido realizados por Salvador Rovira (Museo Arqueológico Nacional, Madrid).
Campa Torres (5) y otro de El Picu Castiellu (6), lo cual indica que al menos en algunos casos, la cámara contaba con una capa de recubrimiento refractario para igualar su superficie y mejorar el rendimiento térmico. El revestimiento de Campa Torres es simple arcilla con la superficie vidriada por efecto del calor. Más interesante y complejo es el revestimiento del horno de El Picu Castiellu, formado por una capa de arena fina, blanca, de unos $8 \mathrm{~mm}$. de espesor, aplicada sobre arcilla. También presenta vidriado y escorificaciones.

La carencia de grandes restos estructurales de hornos motiva que la mayor información provenga del estudio y análisis de las escorias recobradas en zonas de fundición, cuyos resultados se encuentran en la Tabla 1. Como puede comprobarse, las escorias son silicatos de hierro, ligeramente baritadas, y con calcio y/o manganeso en algunas ocasiones, como certifican los análisis de las muestras de Capote (7), Corona de Corporales (8) y Cerro de las Nieves (9). Se comprueba también la existencia de escorias ricas y pobres en hierro, hecho que parece indicar que las condiciones reductoras y el rendimiento en el interior del horno fueron irregulares, como ya había sido observado en las escorias del Morro de Mezquitilla (Keesmann y Hellermann, 1989).

Con el fin de añadir criterios analíticos más precisos a nuestro trabajo, algunas escorias han sido estudiadas con microsonda electrónica,

(5) El castro de La Campa Torres (Gijón) se fecha entre los siglos XV-III a.C. (Maya et alii, 1993: 151). Los materiales estudiados proceden de la estructura denominada "Horno 1".

(6) El Picu Castiellu (Morión, Villaviciosa) es un yacimiento con fases de habitación del Hierro I y II (Camino, 1992). No conocemos la posición estratigráfica de los materiales analizados dentro de dichas fases pero, en todo caso, resultan muy homogéneos.

(7) El Castrejón de Capote (Higuera la Real, Badajoz) es un yacimiento de la Segunda Edad del Hierro excavado por L. Berrocal (1988 y 1993), y donde aparecieron varios elementos relacionados con la fundición de metales tanto de base cobre como de hierro.

(8) La Corona de Corporales (Truchas, León) es un castro prerromano del siglo I a.C. con una importante actividad metalúrgica constatada a través de numerosos hallazgos (Fernández-Posse et alii, 1993).

(9) El Cerro de las Nieves (Pedro Muñoz, Ciudad Real) es un poblado ibérico de los siglos V-IV a.C. (Fernández Martínez et alii. 1994). 


\begin{tabular}{|c|c|c|c|c|c|c|c|c|c|c|c|}
\hline Análisis & Yacimiento & $\mathrm{Fe}$ & $\mathrm{Mn}$ & $\mathrm{Ca}$ & $\mathrm{Ba}$ & $\mathrm{Cu}$ & As & $\mathrm{Sb}$ & $\mathrm{Aq}$ & Sn & $\mathrm{Pb}$ \\
\hline $\begin{array}{l}\text { AA0112 } \\
\text { AA0172 } \\
\text { AA0174 } \\
\text { AA0181 } \\
\text { AA0232 }\end{array}$ & $\begin{array}{l}\text { Llanete de los Moros (Córdoba) } \\
\text { Llanete de los Moros (Córdoba) } \\
\text { Llanete de los Moros (Córdoba) } \\
\text { Llanete de los Moros (Córdoba) } \\
\text { Llanete de los Moros (Córdoba) }\end{array}$ & $\begin{array}{r}113,4 \\
23,9 \\
87,3 \\
27,3 \\
67,7\end{array}$ & $\begin{array}{l}2,0 \\
0,0 \\
0,0 \\
0,0 \\
0,0\end{array}$ & $\begin{array}{l}0,9 \\
0,0 \\
1,9 \\
0,0 \\
0,0\end{array}$ & $\begin{array}{r}9,9 \\
25,2 \\
163,4 \\
30,0 \\
20,7\end{array}$ & $\begin{array}{l}0,0 \\
0,0 \\
0,0 \\
0,0 \\
1,9\end{array}$ & $\begin{array}{l}0,0 \\
0,0 \\
0,0 \\
0,0 \\
0,0\end{array}$ & $\begin{array}{l}0,4 \\
2,3 \\
0,8 \\
0,0 \\
2,8\end{array}$ & $\begin{array}{l}0,0 \\
0,0 \\
0,0 \\
0,0 \\
0,0\end{array}$ & $\begin{array}{l}0,0 \\
0,0 \\
0,6 \\
0,0 \\
1,5\end{array}$ & $\begin{array}{l}0,0 \\
0,0 \\
5,0 \\
0,0 \\
0,0\end{array}$ \\
\hline PA6260 & c/ Puerto, 10 (Huelva) & 35,6 & 0,0 & 1,7 & 14,7 & 0,0 & 0,0 & 0,0 & 0,0 & 0,0 & 0,0 \\
\hline $\begin{array}{l}\text { PA6731 } \\
\text { PA6733 } \\
\text { PA6734 } \\
\text { PA6737 }\end{array}$ & $\begin{array}{l}\text { Castrejón de Capote (Badajoz) } \\
\text { Castrejón de Capote (Badajoz) } \\
\text { Castrejón de Capote (Badajoz) } \\
\text { Castrejón de Capote (Badajoz) }\end{array}$ & $\begin{array}{r}29,7 \\
119,6 \\
151,9 \\
34,6\end{array}$ & $\begin{array}{l}2,2 \\
0,5 \\
1,4 \\
0,0\end{array}$ & $\begin{array}{l}0,8 \\
2,4 \\
0,0 \\
1,0\end{array}$ & $\begin{array}{r}18,4 \\
9,7 \\
13,3 \\
41,6\end{array}$ & $\begin{array}{l}0,0 \\
0,0 \\
0,0 \\
0,0\end{array}$ & $\begin{array}{l}0,0 \\
0,0 \\
0,0 \\
0,0\end{array}$ & $\begin{array}{l}0,8 \\
0,0 \\
0,7 \\
2,0\end{array}$ & $\begin{array}{l}0,0 \\
0,0 \\
0,0 \\
0,0\end{array}$ & $\begin{array}{l}0,0 \\
0,0 \\
0,0 \\
0,0\end{array}$ & $\begin{array}{l}0,0 \\
0,0 \\
0,0 \\
0,0\end{array}$ \\
\hline & & & & & & & & & & & \\
\hline PA3663 & Campa Torres (Asturias) & 111,1 & 0,0 & 0,0 & 25,3 & 0,0 & 0,0 & 0,0 & 0,0 & 1,0 & 0,0 \\
\hline $\begin{array}{l}\text { PA3664 } \\
\text { PA3665 }\end{array}$ & $\begin{array}{l}\text { Campa Torres (Asturias) } \\
\text { Campa Torres (Asturias }\end{array}$ & $\begin{array}{r}98,4 \\
1379\end{array}$ & 0,0 & 0,0 & 62,0 & 0,0 & 0,0 & 0,7 & $\begin{array}{l}0,0 \\
0,0\end{array}$ & ${ }_{12}^{0,6}$ & $\begin{array}{l}0,0 \\
0\end{array}$ \\
\hline PA3666 & Campa Torres (Asturias) & 97,2 & $\begin{array}{l}1,0 \\
0,6\end{array}$ & $\begin{array}{l}0,0 \\
0,0\end{array}$ & 37,3 & $\begin{array}{l}1,2 \\
0,0\end{array}$ & $\begin{array}{l}0,0 \\
0,0\end{array}$ & $\begin{array}{l}0,8 \\
1,0\end{array}$ & 0,0 & 0,0 & 0,0 \\
\hline PA3669 & Campa Torres (Asturias) & 93,5 & 0,0 & 0,0 & 24,1 & 0,0 & 0,0 & 1,1 & 0,0 & 1,0 & 0,0 \\
\hline PA3670 & Campa Torres (Asturias) & 123,9 & 0,0 & 0,0 & 19,8 & 0,0 & 0,0 & 0,1 & 0,0 & 0,5 & 0,0 \\
\hline PA3685-1 & Campa Torres (Asturias) & 15,7 & 0,0 & 0,0 & 56,7 & 0,0 & 0,0 & 0,4 & 0,0 & 1,4 & 0,0 \\
\hline $\begin{array}{l}\text { PA36899 } \\
\text { PA3692 }\end{array}$ & $\begin{array}{l}\text { Campa Torres (Asturias) } \\
\text { Campa Torres (Asturias }\end{array}$ & $\begin{array}{l}32,1 \\
144\end{array}$ & 0,6 & 1,3 & 55,0 & 0,0 & 0,0 & 1,6 & 0,0 & 0,0 & 0,0 \\
\hline PA3693 & $\begin{array}{l}\text { Campa oorres (Asturias) } \\
\text { Campa Torres (Asturias) }\end{array}$ & $\begin{array}{l}14,4 \\
64,0\end{array}$ & $\begin{array}{l}0,0 \\
0,0\end{array}$ & $\begin{array}{l}0,0 \\
0,0\end{array}$ & $\begin{array}{l}51,0 \\
26,1\end{array}$ & $\begin{array}{l}0,0 \\
0,0\end{array}$ & 0,0 & $\begin{array}{l}0,0 \\
0,8\end{array}$ & 0,0 & $\begin{array}{l}0,0 \\
1,2\end{array}$ & 0,0 \\
\hline PA5885 & Campa Torres (Asturias) & 38,0 & 0,0 & 0,0 & 65,1 & 0,0 & 0,0 & 2,5 & 0,0 & 2,0 & 0,0 \\
\hline $\begin{array}{l}\text { PA5886 } \\
\text { PA6249 }\end{array}$ & Campa Torres (Asturias) & 57,2 & 0,0 & 0,0 & 17,1 & 0,0 & 0,0 & 0,0 & 0,0 & 0,0 & 0,0 \\
\hline $\begin{array}{l}\text { PA6249 } \\
\text { PA6250 }\end{array}$ & Campa Torres (Asturias) & 73,8 & 0,0 & 0,0 & 12,2 & 0,0 & 0,0 & 0,0 & 0,0 & 0,0 & 0,0 \\
\hline $\begin{array}{l}\text { PA6250 } \\
\text { PA6251 }\end{array}$ & $\begin{array}{l}\text { Campa Iorres (Asturas) } \\
\text { Campa Torres (Asturias) }\end{array}$ & $\begin{array}{r}10,7 \\
56,4\end{array}$ & $\begin{array}{l}0,0 \\
0,0\end{array}$ & $\begin{array}{l}0,0 \\
0,0\end{array}$ & $\begin{array}{l}1,8 \\
9,0\end{array}$ & $\begin{array}{l}0,0 \\
0,0\end{array}$ & $\begin{array}{l}0,0 \\
0,0\end{array}$ & $\begin{array}{l}0,0 \\
0,0\end{array}$ & $\begin{array}{l}0,0 \\
0,0\end{array}$ & $\begin{array}{l}0,0 \\
0,0\end{array}$ & 0 \\
\hline PA6253 & Campa Torres (Asturias) & 224,4 & 2,7 & 0,0 & 11,6 & 0,0 & 0,0 & 0,0 & 0,0 & 0,0 & 0,0 \\
\hline PA5674 & Carcedo (Asturias) & 240,7 & 4,4 & 0,0 & 13,4 & 0,0 & 0,0 & 0,0 & 0,0 & 0,0 & 0,0 \\
\hline PA5628 & Picu Castiellu (Asturias) & 13,4 & 0,0 & 0,0 & 29,3 & 0,0 & 0,0 & 0,7 & 0,0 & 0,8 & 0,0 \\
\hline $\begin{array}{l}\text { PA5629 } \\
\text { PA5630 }\end{array}$ & $\begin{array}{l}\text { Picu Castiellu (Asturias) } \\
\text { Picu Castiellu (Asturias }\end{array}$ & 39,1 & 0,0 & 0,0 & 25,5 & 0,0 & 0,0 & 0,4 & 0,0 & 0,3 & 0,0 \\
\hline $\begin{array}{l}\text { PA5630 } \\
\text { PA5632-1 }\end{array}$ & $\begin{array}{l}\text { Picu Castiellu (Asturias) } \\
\text { Pisule (Asturiastu }\end{array}$ & 176,2 & 0,0 & 0,0 & 11,7 & 0,0 & 0,0 & 0,4 & 0,0 & 0,2 & 0,0 \\
\hline PA5632-2 & $\begin{array}{l}\text { Picu Castiellu (Asturias) } \\
\text { Picu Castiellu (Asturias) }\end{array}$ & $\begin{array}{r}17,8,8 \\
28,2\end{array}$ & $\begin{array}{l}0,0 \\
0,0\end{array}$ & $\begin{array}{l}0,0 \\
0,0\end{array}$ & $\begin{array}{r}0,9 \\
17,3\end{array}$ & $\begin{array}{l}0,0 \\
0,0\end{array}$ & $\begin{array}{l}0,0 \\
0,0\end{array}$ & 0,1 & 0,0 & $\begin{array}{l}0,2 \\
0,3\end{array}$ & $\begin{array}{l}0,0 \\
0,0\end{array}$ \\
\hline PA5632-3 & Picu Castiellu (Asturias) & 171,7 & 0,0 & 0,0 & 5,5 & 0,0 & 0,0 & 0,3 & 0,0 & 0,0 & 0,0 \\
\hline $\begin{array}{l}\text { PA5632-4 } \\
P A 533-1\end{array}$ & $\begin{array}{l}\text { Picu Castiellu (Asturias) } \\
\text { Picu }\end{array}$ & 194,8 & 0,0 & 0,0 & 1,0 & 0,0 & 0,0 & 0,4 & 0,0 & 0,3 & 0,0 \\
\hline $\begin{array}{l}\text { PA5633-1 } \\
\text { PA5633-2 }\end{array}$ & $\begin{array}{l}\text { Picu Castiellu (Asturias) } \\
\text { Picullu (Asturias }\end{array}$ & $\begin{array}{l}72,9 \\
874\end{array}$ & 0,0 & 0,0 & 32,0 & 0,0 & 0,0 & 0,8 & 0,0 & 0,5 & 0,0 \\
\hline $\begin{array}{l}\text { PA5633--2 } \\
\text { PA563-3 }\end{array}$ & $\begin{array}{l}\text { Picu Castelellu (Asturass) } \\
\text { Picu Castiellu (Asturias) }\end{array}$ & $\begin{array}{r}8,4 \\
7,4\end{array}$ & $\begin{array}{l}0,0 \\
0,0\end{array}$ & $\begin{array}{l}0,0 \\
0,0\end{array}$ & $\begin{array}{l}21,3 \\
12,2\end{array}$ & $\begin{array}{l}0,0 \\
0,0\end{array}$ & $\begin{array}{l}0,0 \\
0,0\end{array}$ & 0,4 & $\begin{array}{l}0,0 \\
0,0\end{array}$ & $\begin{array}{l}0,8 \\
0,8\end{array}$ & $\begin{array}{l}0,0 \\
0,0\end{array}$ \\
\hline PA5633-4 & Picu Castiellu (Asturias) & 251,9 & 0,0 & 0,0 & 1,6 & 0,0 & 0,0 & 0,3 & 0,0 & 0,1 & 0,0 \\
\hline PA5634-1 & Picu Castiellu (Asturias) & 179,8 & 0,0 & & 5,7 & 0,0 & 0,0 & 0,1 & 0,0 & 0,2 & 0,0 \\
\hline PA5634-2 & Picu Castiellu (Asturias) & 24,6 & 0,0 & 0,0 & 29,2 & & 0,0 & 0,3 & 0,0 & 0,5 & 0,0 \\
\hline $\begin{array}{l}\text { PA5635-1 } \\
P A 565-2\end{array}$ & 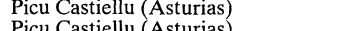 & $\begin{array}{l}180,2 \\
265\end{array}$ & 0,0 & 0,0 & $\begin{array}{r}8,4 \\
35,1\end{array}$ & 0,0 & 0,0 & 0,3 & 0,0 & 0,1 & 0,0 \\
\hline PA5666A & Picu Castiellu (Asturias) & $\begin{array}{r}21,3 \\
113,6\end{array}$ & ${ }_{0,0}^{0,0}$ & $\begin{array}{l}0,0 \\
0,0\end{array}$ & $\begin{array}{r}35,1 \\
0,0\end{array}$ & $\begin{array}{l}0,0 \\
0,0\end{array}$ & 0,0 & 0,4 & 0,0 & 0,0 & 0,0 \\
\hline PA5666B & Picu Castiellu (Asturias) & 77,1 & 0,0 & 0 & $\begin{array}{l}0,0 \\
33,0\end{array}$ & 0,0 & 0,0 & 0,0 & 0,0 & $\begin{array}{l}0,4 \\
0,0\end{array}$ & 0,0 \\
\hline PA5667-1 & Picu Castiellu (Asturias) & 237,1 & 0,0 & 0,0 & 4,7 & 0,0 & 0,0 & 0,3 & 0,0 & 0,0 & 0,0 \\
\hline PA5667-2 & Picu Castiellu (Asturias) & 194,5 & 0,0 & 0,0 & 5,4 & 0,0 & 0,0 & 0,2 & 0,0 & 0,2 & 0,0 \\
\hline PA5667-3 & Picu Castiellu (Asturias) & 243,5 & 0,0 & 0,0 & 2,2 & 0,0 & 0,0 & 0,3 & 0,0 & 0,0 & 0,0 \\
\hline PA5668 & Picu Castiellu (Asturias) & 177,6 & 0,0 & 0,0 & 8,2 & 0,0 & 0,0 & 0,0 & 0,0 & 0,0 & 0,0 \\
\hline PA5669 & Picu Castiellu (Asturias) & 174,9 & 0,0 & 0,0 & 7,1 & 0,0 & 0,0 & 0,3 & 0,0 & 0,2 & 0,0 \\
\hline $\begin{array}{l}\text { PA5672 } \\
\text { PA5678 }\end{array}$ & $\begin{array}{l}\text { Picu Castiellu (Asturias) } \\
\text { Picu Castiellu (Asturias) }\end{array}$ & $\begin{array}{l}219,4 \\
178\end{array}$ & 0,0 & 0,0 & 8,1 & 0,0 & 0,0 & 0,4 & 0,0 & 0,0 & 0,0 \\
\hline PA6268 & $\begin{array}{l}\text { Picu Castelllu (Asturias) } \\
\text { Picu Castiellu (Asturias) }\end{array}$ & $\begin{array}{l}178,8 \\
171,7\end{array}$ & $\begin{array}{l}0,0 \\
0,0\end{array}$ & $\begin{array}{l}0,0 \\
0,0\end{array}$ & $\begin{array}{r}8,5 \\
10,5\end{array}$ & $\begin{array}{l}0,0 \\
0,0\end{array}$ & $\begin{array}{l}0,0 \\
0,0\end{array}$ & $\begin{array}{l}0,3 \\
0,4\end{array}$ & $\begin{array}{l}0,0 \\
0,0\end{array}$ & $\begin{array}{l}0,0 \\
0,0\end{array}$ & $\begin{array}{l}0,0 \\
0,0\end{array}$ \\
\hline PA0439 & Corona de Corporales (León) & 182,0 & 2,8 & 0,0 & 1,8 & 0,0 & 0,0 & 0,5 & 0,0 & 0,0 & 0,0 \\
\hline PA0440A & Corona de Corporales (León) & 139,7 & 2,4 & 0,0 & 5,3 & 1,2 & 0,0 & 0,9 & 4,2 & 0,0 & 0,0 \\
\hline PA0440B & Corona de Corporales (León) & 123,8 & 0,0 & 0,0 & 3,5 & 0,0 & 0,0 & 0,7 & 3,5 & 0,0 & 0,0 \\
\hline $\begin{array}{l}\text { PA0440C } \\
\text { PA0440D1 }\end{array}$ & Corona de Corporales (León) & $\begin{array}{l}47,7 \\
356\end{array}$ & $\begin{array}{l}3,7 \\
3,7\end{array}$ & 0,0 & 43,4 & 0,0 & 0,0 & 0,8 & 1,0 & 0,5 & 0,0 \\
\hline PA0440D2 & $\begin{array}{l}\text { Corona de Corporales (Leon) } \\
\text { Coronales (León }\end{array}$ & $\begin{array}{l}35,6 \\
14,4\end{array}$ & $\begin{array}{l}3,7 \\
0,9\end{array}$ & $\begin{array}{l}0,0 \\
0,0\end{array}$ & $\begin{array}{l}66,1 \\
23,7\end{array}$ & $\begin{array}{l}0,0 \\
0,0\end{array}$ & $\begin{array}{l}0,0 \\
0,0\end{array}$ & $\begin{array}{l}0,4 \\
1,7\end{array}$ & $\begin{array}{l}0,6 \\
1,0\end{array}$ & 0,4 & 0,0 \\
\hline PA0440D3 & Corona de Corporales (León) & 178,3 & 1,1 & 0,0 & 4,0 & 0,0 & 0,0 & 0,4 & 1,1 & 0,0 & 0,0 \\
\hline PA0440A1 & Corona de Corporales (León) & 16,1 & 0,0 & 0,0 & 31,8 & 0,0 & 0,0 & 1,3 & 1,7 & 0,0 & 0,0 \\
\hline $\begin{array}{l}\text { PA0441A2 } \\
\text { PA0441B }\end{array}$ & Corona de Corporales (León) & $\begin{array}{l}50,9 \\
668\end{array}$ & 4,5 & 0,0 & 31,4 & 0,0 & 0,0 & 0,3 & 0,7 & 1,6 & 0,0 \\
\hline $\begin{array}{l}\text { PAA4411B } \\
\text { PA042B }\end{array}$ & $\begin{array}{l}\text { Corona de Corporales Leonn) } \\
\text { Corona de Corporales (León) }\end{array}$ & $\begin{array}{r}62,8 \\
123,5\end{array}$ & ${ }_{2,1}^{1,6}$ & $\begin{array}{l}0,0 \\
0,0\end{array}$ & $\begin{array}{r}21,3 \\
7,7\end{array}$ & $\begin{array}{l}0,0 \\
0,0\end{array}$ & $\begin{array}{l}0,0 \\
0,0\end{array}$ & $\begin{array}{l}0,0 \\
0,9\end{array}$ & $\begin{array}{l}0,2 \\
0,0\end{array}$ & $\begin{array}{l}0,4 \\
0,0\end{array}$ & 0,0 \\
\hline PA0443A & & 86,3 & 0,0 & 0,0 & 13,6 & 0,0 & 0,0 & 0,3 & 2,3 & 0,7 & 0,0 \\
\hline PA0443B & Corona de Corporales (León) & 56,2 & 2,0 & 0,0 & 29,3 & 0,0 & 0,0 & 0,0 & 0,0 & 0,0 & 0,0 \\
\hline $\begin{array}{l}\text { PA0443C } \\
\text { PA0444 }\end{array}$ & Corona de Corporales (León) & $\begin{array}{l}30,4 \\
981\end{array}$ & 3,6 & 0,0 & 41,4 & 0,0 & 0,0 & 0,0 & 0,0 & 0,0 & 0,0 \\
\hline $\begin{array}{l}\text { PA0444 } \\
\text { PA445A }\end{array}$ & $\begin{array}{l}\text { Corona a de corporales (Leon) } \\
\text { Corona de Corporales (León) }\end{array}$ & $\begin{array}{l}98,1 \\
57,6\end{array}$ & $\begin{array}{l}2,9 \\
5,8\end{array}$ & $\begin{array}{l}0,0 \\
0,0\end{array}$ & $\begin{array}{l}16,8 \\
44,7\end{array}$ & $\begin{array}{l}0,0 \\
0,0\end{array}$ & $\begin{array}{l}0,0 \\
0,0\end{array}$ & $\begin{array}{l}0,4 \\
0,0\end{array}$ & $\begin{array}{l}0,4 \\
0,0\end{array}$ & $\begin{array}{l}0,2 \\
0,0\end{array}$ & 0,0 \\
\hline PA0445B & & 53,0 & 6,6 & 0,0 & 46,9 & 0,0 & 0,0 & 0,0 & 0,0 & 0,0 & 0,0 \\
\hline PA0447A & Corona de Corporales (León) & 178,7 & 1,8 & 0,0 & 1,4 & 0,0 & 0,0 & 0,0 & 0,0 & 0,0 & 0,0 \\
\hline $\begin{array}{l}\text { PA0447B } \\
\text { PA0448 }\end{array}$ & $\begin{array}{l}\text { Corona de Corporales (León) } \\
\text { Corona de Corporales Leoón }\end{array}$ & $\begin{array}{r}211,7 \\
281\end{array}$ & 2,2 & 0,0 & $\begin{array}{r}1,4 \\
514\end{array}$ & 0,0 & 0,0 & 0,0 & 0,0 & 0,0 & 0,0 \\
\hline PA0451A & $\begin{array}{l}\text { Corona de Corporales es (León) } \\
\text { Corona de }\end{array}$ & $\begin{array}{r}28,1 \\
160,2\end{array}$ & $\begin{array}{l}5,2 \\
0,0\end{array}$ & $\begin{array}{l}0,0 \\
0,0\end{array}$ & $\begin{array}{r}1,4 \\
0,4\end{array}$ & $\begin{array}{l}0,0 \\
0,0\end{array}$ & $\begin{array}{l}0,0 \\
0,0\end{array}$ & 0,5 & $0,0,0$ & 0,0 & 0,0 \\
\hline PA0451B & Corona de Corporales (León) & 191,6 & 0,0 & 0,0 & 0,2 & 0,0 & 0,0 & 0,4 & 0,2 & 0,0 & 0,0 \\
\hline $\begin{array}{l}\text { PA0452 } \\
\text { PA0453A }\end{array}$ & $\begin{array}{l}\text { Corona de Corporales (León) } \\
\text { Corona de Corporales (León) }\end{array}$ & $\begin{array}{l}44,1 \\
18,8\end{array}$ & $\begin{array}{l}4,8 \\
1,8\end{array}$ & $\begin{array}{l}0,0 \\
0,0\end{array}$ & $\begin{array}{l}45,4 \\
21,3\end{array}$ & $\begin{array}{l}0,0 \\
0,0\end{array}$ & $\begin{array}{l}0,0 \\
0,0\end{array}$ & $\begin{array}{l}0,8 \\
1,4\end{array}$ & $\begin{array}{l}0,0 \\
7,7\end{array}$ & $\begin{array}{l}0,0 \\
0,0\end{array}$ & $\begin{array}{l}0,0 \\
0,0\end{array}$ \\
\hline A3708-6 & El Amarejo (Albacete) & 95,5 & 0,6 & 0,9 & 3,8 & 0,3 & 0,0 & 0,8 & 0,0 & 0,0 & 0,0 \\
\hline PA6513 & El Cerro de las Nieves (Ciudad & 45,6 & 0,0 & & & 0,0 & 0,0 & 2,5 & 0,0 & 1,6 & 0,0 \\
\hline PA6514 & El Cerro de las Nieves (Ciudad Real) & 230,7 & 0,0 & 1,4 & 3,6 & 0,0 & 0,0 & 0,0 & 0,0 & 0,0 & 0,0 \\
\hline PA6515 & El Cerro de las Nieves (Ciudad Real) & 178,2 & 0,0 & 0,0 & 5,0 & 0 & 0,0 & 0,0 & 0,0 & 0,0 & 0,0 \\
\hline $\begin{array}{l}\text { PA6518 } \\
\text { PA6510 }\end{array}$ & El Cerro de las Nieves (Ciudad Real) & 136,3 & 0,0 & 3,1 & 3,0 & 0,0 & 0,0 & 0,0 & 0,0 & 0,0 & 0,0 \\
\hline $\begin{array}{l}\text { PA6519 } \\
\text { PA6520 }\end{array}$ & $\begin{array}{l}\text { EI Cerro de las Nieves (Ciudad Real) } \\
\text { El Cerro de las Nieves (Ciudad Real) }\end{array}$ & $\begin{array}{l}24,8 \\
56,3\end{array}$ & ${ }_{0,0}^{1,6}$ & 2,8 & $\begin{array}{r}35,4 \\
57\end{array}$ & 0,0 & $\begin{array}{l}0,0 \\
0,0\end{array}$ & $\begin{array}{l}0,0 \\
0,0\end{array}$ & $\begin{array}{l}0,0 \\
0\end{array}$ & $\begin{array}{l}0,0 \\
0,0\end{array}$ & $\begin{array}{l}0,0 \\
0,0\end{array}$ \\
\hline PA6536 & El Cerro de las Nieves (Ciudad Real) & $\begin{array}{l}58,3 \\
88,2\end{array}$ & $\begin{array}{l}0,0 \\
0,0\end{array}$ & $\begin{array}{l}4,1 \\
1,5\end{array}$ & 3,9 & 0,0 & 0,0 & 0,0 & $\begin{array}{l}0,0 \\
0,0\end{array}$ & 0,0 & 0,0 \\
\hline
\end{tabular}

Tabla 1. Análisis espectral de escorias siderúrgicas (resultados en cuentas/segundo).

T. P., 53, n. ${ }^{\circ} 2,1996$ 
por fluorescencia de rayos $\mathrm{X}$, en el microscopio de barrido. Así, la muestra PA3670 de Campa Torres, que en la Tabla 1 da un pico de hierro de 123,9 c.p.s., contiene un $78,6 \%$ de óxido férrico, lo que equivale a un contenido de este elemento del $54,97 \%$. Es, por tanto, una escoria rica en metal. Su contenido en sílice es del $21,41 \%$. Por el contrario, la muestra PA6731 de Capote es una escoria pobre en hierro, con 29,7 c.p.s., pico que se corresponde con una cifra total del $12 \%$ de hierro. El contenido de sílice es aquí del 71,33\%.

Los estudios microscópicos corroboran también procesos poco eficientes para la reducción de los minerales de hierro. Se lograba formar escorias fayalíticas (10), pero el ambiente del horno no acababa de ser el adecuado para reducir todo el hierro presente, por lo que gran parte de las escorias suele ser muy rica en óxidos de hierro (wustita y magnetita) y presenta propiedades ferromagnéticas (Lám. I A y B). Es frecuente, igualmente, encontrar pequeños nódulos de hierro metálico en la escoria (Lám. I C), lo que indica una precaria tecnología del horno por las pérdidas de hierro que comportan, aunque es probable que tales escorias fueran reutilizadas en una fundición posterior, como ya señalaran Keesmann et alii (1989: 107) cuando estudiaron materiales similares hallados en Toscanos. La presencia de sílice libre es, por otro lado, signo evidente del empleo de arena como fundente, como se ha podido comprobar en El Picu Castiellu, Campa Torres y Cerro de las Nieves. Es normal observar granos de cuarzo intactos que no lograron fundirse ya que las temperaturas no fueron lo suficientemente altas, pero indican en todo caso un procedimiento correcto como es la adición de fundentes silícicos en la obtención de hierro.

Estos materiales indican que la producción de hierro se efectuaba en pequeños hornos de morfología no determinada aunque, eso sí, sin sangrado de escorias, lo que conduce a la for-

(10) La fayalita es un silicato de hierro que cristaliza en la escoria formando cristales aciculares. Funde en torno a los $1.100^{\circ} \mathrm{C}$, por lo que su presencia y abundancia son indicadores de la temperatura de formación de la escoria. La fayalita de la muestra PA6733 de Capote contiene 26,05\% de sílice y $70,95 \%$ de óxido ferroso.

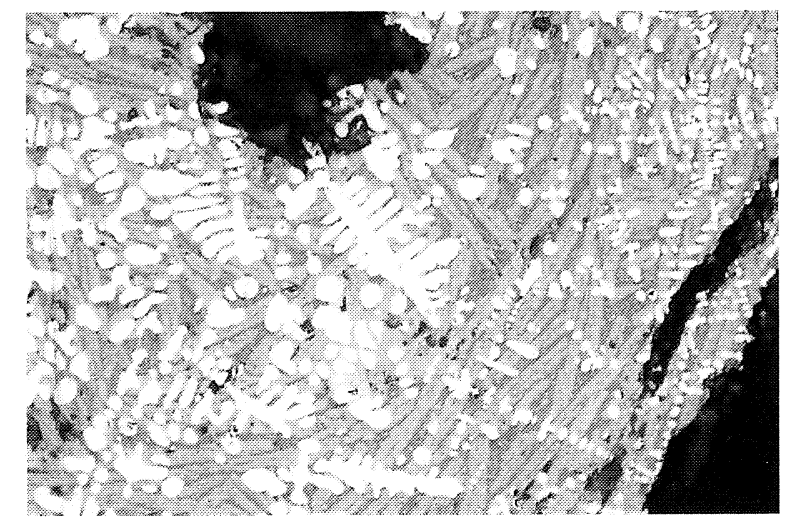

A

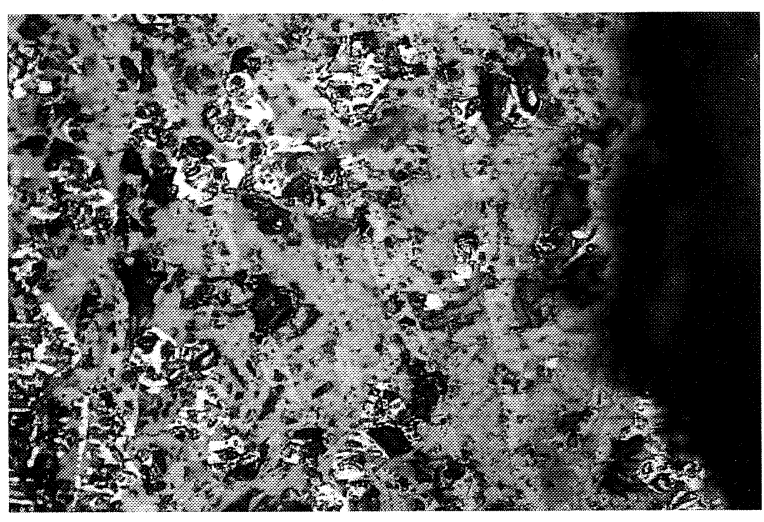

B

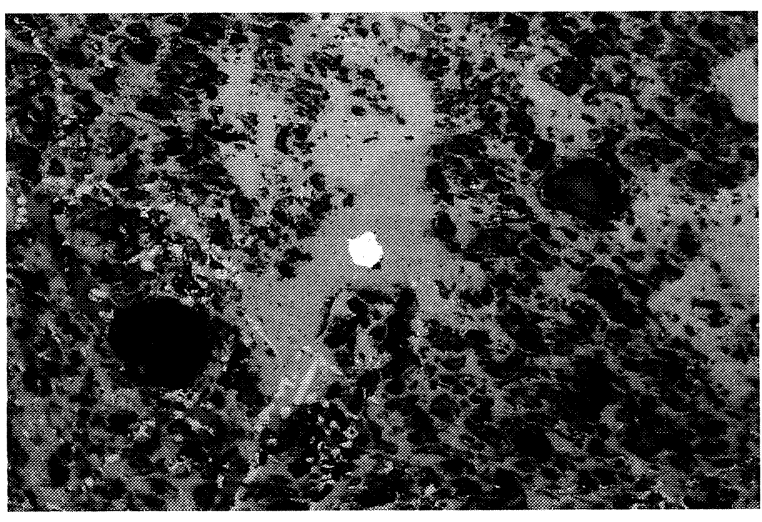

C

Lám. I: A: Microfotografía de la escoria PA5677-1 de El Picu Castiellu (Asturias). Fayalita acicular (color gris claro) y wustita dendrítica (color blanco) en una matriz de vidrio de relleno (color gris oscuro). Campo claro. 200x. B: Microfotografía de la escoria PA3689 de La Campa Torres (Asturias). Zona fayalítica con formaciones blancas de magnetita. Campo claro. 250x. C: Microfotografía de la escoria PA3670 de La Campa Torres (Asturias). En el centro de la imagen se observa un glóbulo blanco de hierro metálico. Campo claro. $250 \mathrm{x}$. 


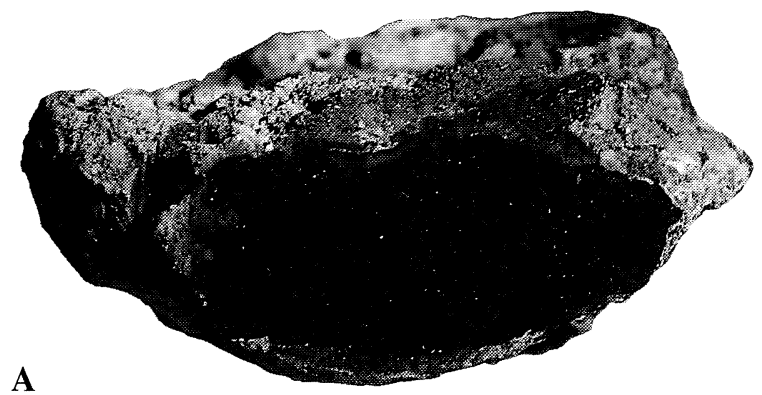

A

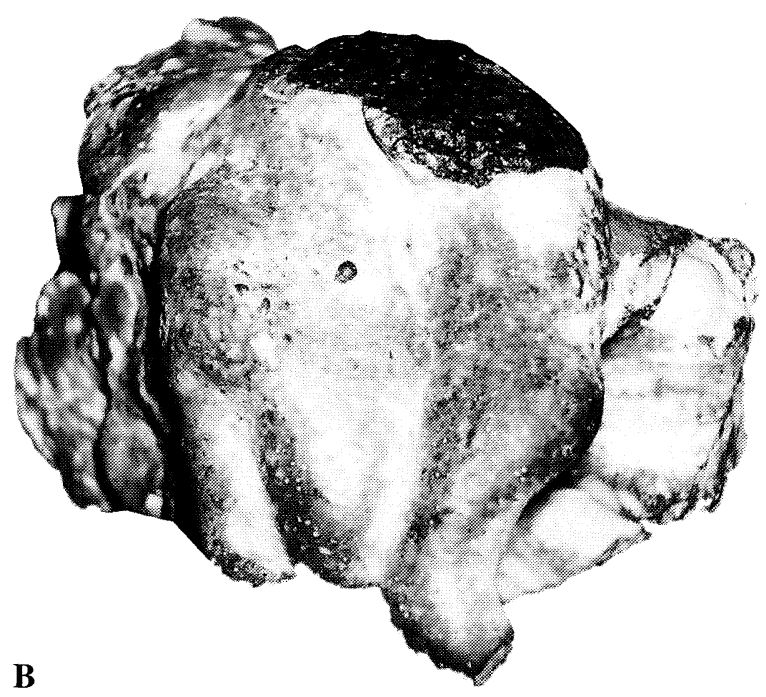

Lám. II: A: Torta plano-convexa de escoria de hierro PCM 2411 de El Picu Castiellu (Asturias), de unos 12 $\mathrm{cm}$. de diámetro. Obsérvese el aspecto poroso de la sección. B: Aspecto externo de un pequeño fragmento de la escoria de sangrado PA6733 de Capote (Badajoz), $7 \mathrm{~cm}$.

mación de las características tortas de forma planoconvexas depositadas en el fondo del horno, ya que, a diferencia del trabajo del cobre-bronce, donde el metal se depositaba en el fondo del horno, en la reducción de hierro es la escoria la que cae a la solera de la estructura, mientras que la lupia o esponja férrica se forma más arriba, cerca del flujo de las toberas, que es la zona donde se alcanzan las mayores temperaturas. Se han encontrado escorias de horno en Picu Castiellu (Lám. II A), el Cerro de las Nieves o el Castrejón de Capote. Suelen ser piezas de forma concoide, con superficies rugosas y oxidaciones ferruginosas. Del Castrejón de Capote y también de Carce- do (11) hemos estudiado un fragmento de escoria de sangrado (Lám. II B), lo que podría evidenciar la introducción de esta mejora del horno, al menos en estos dos sitios. En el caso de Carcedo el testimonio proviene de prospecciones, por lo que no es fácil establecer su correspondencia cronológica exacta. No ocurre lo mismo con la escoria de Capote, yacimiento excavado con meticulosidad. Se halló en un contexto cerrado relacionado con el Altar prerromano, de un momento avanzado de la II Edad del Hierro (Berrocal, 1994), por lo que se trata, hasta la fecha, de un unicum que podría indicar la utilización de hornos de mayor capacidad.

\section{CONCLUSIONES}

Con la adopción de la siderurgia, la Península Ibérica ingresa plenamente en la Edad del Hierro. La presencia de este nuevo metal, que ampliará notablemente las posibilidades de la producción metalúrgica, se atribuye a los contactos establecidos con fenicios y griegos a través de sus colonias más tempranas en Occidente (Snodgrass, 1980). Los restos más antiguos de estructuras de hornos de hierro en la Península, de los cuales contamos con mayor información, se limitan a los hallazgos murcianos de El Castellar de Librilla, fechados en los siglos VIII-VII a.C. (Ros Sala, 1989 y 1993), y a Toscanos, Málaga, a mediados del siglo VII a.C. (Keesmann et alii, 1989). Las escorias no son de sangrado, lo cual indicaría cierto primitivismo de estos hornos, más sorprendente si consideramos que la metalurgia de Toscanos y también la de El Morro de Mezquitilla es la de dos asentamientos fenicios. Pero al carecer de datos sobre los hornos sirio-palestinos contemporáneos, tal primitivismo no deja de ser una impresión apriorística probablemente errónea, construida tras aceptar sin crítica que los fenicios debían conocer una tecnología mejor.

Efectivamente, si bien no hay por el momento datos de la tecnología siderúrgica de la me-

(11) Yacimiento asturiano de época prerromana y romana, inédito, prospectado por Jorge Camino Mayor (arqueólogo del Principado de Asturias). 
trópolis fenicia, sí tenemos algunos, aunque más tardíos, de localidades italianas que nos pueden servir de ilustración acerca del tema. Así, por ejemplo, en Populonia (Toscana) se encontraron restos de un horno cilíndrico de unos $30 \mathrm{~cm}$. de diámetro interior y unos $45 \mathrm{~cm}$. de altura fechado en el $420 \pm 100 \mathrm{ca}$. AC. Este horno se halla en un área de gran actividad siderúrgica, como demuestran los miles de toneladas de escorias entre los que se encuentra (aunque sólo una parte es de época etrusca, pues la mayor parte son de época romana y medieval). La escoria producida en este horno no es de sangrado y consiste en pequeños nódulos actualmente cementados formando capas en la playa de $\mathrm{Ba}-$ ratti. En este mismo sitio se documentan grandes tortas de escoria de sangrado asociadas a hornos fechados en el $170 \pm 70 \mathrm{ca}$. AC. cuyos restos responden a estructuras cilíndricas de aproximadamente un metro de altura y $50 \mathrm{~cm}$. de diámetro interior en la solera (Voss, 1988).

Así, pues, si estos hornos mediterráneos son como acabamos de describir y con ellos se obtuvieron miles de kilogramos de hierro, no hay ninguna razón para pensar que los hornos fenicios de Toscanos y de El Morro de Mezquitilla, más antiguos, tuvieran que ser mayores y más evolucionados, cuando en ninguna factoría colonial se ha podido documentar un volumen de escorias ni siquiera mediano que los justifique.

Todo parece señalar que la actividad siderúrgica hispánica fue muy limitada dada la inexistencia de buenos escoriales, pero la tecnología puesta en juego era tan moderna o si se prefiere, tan atrasada, como la de otras regiones mediterráneas. Nuestros análisis de escorias, coincidentes con los de otros investigadores, así lo corroboran.

Dentro de este panorama general en el que no se observan diferencias significativas en la tecnología siderúrgica de distintas partes de Europa, encajan sin dificultad los datos aportados por nuestra investigación. Todos los fragmentos de escorias que hemos estudiado son de hornos sin sangrado con la excepción de una única muestra del Castrejón de Capote, probablemente moderna dentro del Hierro II, pero que por su carácter singular no conviene sobrevalorar. Las tortas de escoria de El Picu Castiellu, Capote, Cerro de las Nieves, etc., se forma- ron en el fondo de pequeños hornos de no más de $30-40 \mathrm{~cm}$. de diámetro, si bien lo más normal es encontrar escorias en forma de agregados nodulares, característicos también de estos hornos sencillos cuya morfología no conocemos en detalle, pero que podían llevar revestimientos internos ricos en sílice. En los hornos se procesaban óxidos de hierro, utilizando como fundente arena. Sin embargo, las condiciones reductoras no eran siempre las más apropiadas, lo que motivaba con frecuencia la formación de magnetita. No será hasta época romana cuando la metalurgia extractiva del hierro alcance rasgos más evolucionados de la mano de hornos con sangrado de escorias de tipo fayalítico.

En cualquier caso, el Hierro Antiguo es una etapa de nuestra Protohistoria que debe ser estudiada con más meticulosidad por lo que respecta a la metalurgia férrica, ya que muchos de los materiales aquí presentados carecen de precisa base cronológica. No obstante, no se observan grandes diferencias morfológicas ni químicas en el conjunto de las escorias, lo cual hace pensar que probablemente no encontraremos contrastes apreciables entre las tecnologías del Hierro I y II, sino una mayor extensión e intensificación del empleo del hierro. La escoria de sangrado de Capote podría interpretarse, no tanto como una mejora del horno sino como un aumento de la producción del mismo.

En la Península Ibérica no se han documentado por ahora grandes acumulaciones de escorias de hierro con las que sustentar la tesis de la existencia de algún gran centro productor prerromano. Por el contrario, la frecuencia de sitios arqueológicos con pequeños restos de esta actividad, juntơ con la diseminación y abundancia de las mineralizaciones ferruginosas, remiten a una práctica siderúrgica a pequeña escala, local, dedicada al autoabastecimiento. Quizá por ello no se registran, a diferencia de lo que sucede en otras partes de Europa, hallazgos claros de lingotes de hierro, pues el ciclo se inicia y acaba en el propio taller del herrero.

\section{BIBLIOGRAFÍA}

Almagro-Gorbea, M. (1993): "La introducción del hierro en la Península Ibérica. Contactos precoloniales 
en el período protoorientalizante". Complutum, 4: 81-94.

Arana, R. y Pérez Sirvent, C. (1993): "El trabajo del hierro en el poblado protohistórico de El Castellar de Librilla (Murcia). II. Estudio mineralógico". En R. Arana, A.M ${ }^{a}$. Muñoz, S. Ramallo y M. Ros (eds.): $M e-$ talurgia en la Península Ibérica durante el primer milenio a.C. Estado actual de la investigación. Universidad de Murcia. Murcia: 111-129.

Berrocal, L. (1988): Excavaciones en Capote (Beturia céltica). I. Serie Nertobriguense, 1. Badajoz.

- (1993): Los pueblos célticos del Suroeste de la Península Ibérica. Complutum Extra, 2. Universidad Complutense. Madrid.

- (1994): El Altar prerromano de Capote. Ensayo etnoarqueológico de un ritual céltico en el Suroeste peninsular. Universidad Autónoma. Madrid.

CAmino, J. (1992): "Excavaciones arqueológicas en castros de la Ría de Villaviciosa: un poblamiento de la Edad del Hierro". Excavaciones arqueológicas en Asturias 1987-90: 137-144.

Cleere, H. (1972): "The classification of early iron-smelting furnaces". The Antiquaries Journal, LII: 8-23.

Coghlan, H. (1977): Notes on Prehistoric and Early Iron in the Old World. Pitt Rivers Museum. Oxford.

Esparza Arroyo, A. (1987): Los Castros de la Edad del Hierro del Noroeste de Zamora. Instituto de Estudios Zamoranos "Florián de Ocampo" y la Diputación de Zamora. Zamora.

Fernández Martínez, V.; Hornero, E. y Pérez MugA, J. (1994): "El poblado ibérico del Cerro de las Nieves (Pedro Muñoz). Excavaciones 1984-1985". Jornadas de Arqueología de Ciudad Real en la Universidad Autónoma de Madrid. Patrimonio HistóricoArqueología de Castilla-La Mancha. Madrid: 111129.

Fernández-Posse, Ma.D.; Montero, I.; Sánchez PalenCIA, J. y RovirA, S. (1993): "Espacio y metalurgia en la cultura castreña: la zona arqueológica de las Médulas". Trabajos de Prehistoria, 50: 197-220.

Forbes, R.J. (1972): Studies in Ancient Technology. IX. E. J. Brill. Leiden. $2^{\text {a }}$ ed. revisada.

Gómez Ramos, P. (1996)a: "Hornos de reducción de cobre y bronce en la Pre y Protohistoria de la Península Ibérica". Trabajos de Prehistoria, 53 (1): 127-143.

- (1996)b: La tecnología de fundición de metales en la Pre y Protohistoria de la Península Ibérica. Tesis Doctorales microfichadas de la Universidad Autónoma. Madrid.

Hernández Vera, J. y Murillo Ramos, J. (1985): "Aproximación al estudio de la siderurgia celtibérica del Moncayo". Caesaraugusta, 61-62: 177-190.

KeEsmanN, I. y HellermanN, B. (1989): "Mineralogische und chemische untersuchungen an schlacken vom Morro de Mezquitilla". Madrider Mitteilungen, 30: 92117.

Keesmann, I.; Niemeyer, H.; Briese, C.; Golschani, F. y Schulz, B. (1989): "Un centro primitivo de la elaboración de hierro en la factoría fenicia de Toscanos". En C. Domergue (coord.): Coloquio Internacional sobre
Minería y Metalurgia en las antiguas civilizaciones mediterráneas y europeas. I. Ministerio de Cultura. Madrid: 99-108.

Madroñero, A.; Martín Costea, A.; López Serrano, V.; García CARcedo, F. y Arlegui, M. (1992): "Estudio arqueometalúrgico de útiles y restos minerometalúrgicos de hierro del yacimiento celtibérico de "Castilmontán" (Somaén, Soria)". Museo de Zaragoza. Boletín, 11: 47-88.

Martín Costea, A. y Ruiz Zapatero, G. (1980): "La metalurgia del hierro en el poblado protohistórico de Vallipón (Teruel)". Revista de Metalurgia, 37: 31-40.

Martín Costea, A.; Madroñero, A.; LóPez Serrano, V. y GARCÍA CARCEDO, F. (1993): "Arqueometalurgia del poblado ibérico Monte Catma (La Ginebrosa, Teruel)". Grupo de Estudios Masinos, 13: 241-283.

MAYA, J.; Rovira, S. y Cuesta, F. (1993): "Metalurgia del bronce en el poblado prerromano de La Campa Torres (Asturias)". Pyrenae, 24: 151-158.

NiemeYer, H.G. (1982): "El yacimiento fenicio de Toscanos: balance de la investigación 1964-1979". Primeras Jornadas Arqueológicas sobre colonizaciones orientales. Huelva Arqueológica, VI: 101-130.

Ró Sala, M. (1989): Dinámica urbanística y cultura material del Hierro Antiguo en el valle del Guadalentín. Colegio Oficial de Arquitectos de Murcia y la Universidad de Murcia. Murcia.

- (1993): "El trabajo del hierro en el poblado protohistórico de El Castellar (Murcia). I: análisis arqueológico". En R. Arana, A.M ${ }^{a}$. Muñoz, S. Ramallo y M. Ros (eds.): Metalurgia en la Península Ibérica durante el primer milenio a.C. Estado actual de la investigación. Universidad de Murcia. Murcia: 71-109.

SCHUBART, H. (1985)a: "Morro de Mezquitilla. Informe preliminar sobre la campaña de excavaciones de 1982 realizada en el asentamiento fenicio cerca de la desembocadura del río Algarrobo". Noticiario Arqueológico Hispánico, 23: 141-174.

- (1985)b: "El asentamiento fenicio del siglo VIII a.C. en el Morro de Mezquitilla (Algarrobo, Málaga)”. Aula Orientalis, III: 59-83.

- (1990): "Los primeros asentamientos fenicios en las costas de la Península Ibérica". Archivo de Prehistoria Levantina, Homenaje a D. Enrique Pla Ballester. XX: 29-41.

Schubart, H. y ARTEga, O. (1986): "El mundo de las colonias fenicias occidentales". Actas del Congreso "Homenaje a Luis Siret" (1934-1984) (Cuevas del Almanzora, junio de 1984). Consejería de Cultura de la Junta de Andalucía. Madrid: 499-525.

SCHUBART, H. y MAAss-LindeMANN, G. (1984): "Toscanos. El asentamiento fenicio occidental en la desembocadura del río de Vélez. Excavaciones de 1971". Noticiario Arqueológico Hispánico, 18: 39-210.

SChUbART, H. y NiEMEYeR, H. G. (1969): "La factoría paleopúnica de Toscanos (resultados de las excavaciones estratigráficas)". Tartessos y sus problemas. V Symposium internacional de Prehistoria Peninsular. Jerez de la Frontera. Septiembre de 1968. Universidad de Barcelona. Barcelona: 203-219.

T. P., 53, n. ${ }^{\circ} 2,1996$ 
SNODGRASS, A. (1980): "Iron and Early Metallurgy in the Mediterranean". En T. Wertime y J. Muhly (eds.): The Coming of the Age of Iron. Yale University. New Haven: 335-374.

TyleCOTE, R. (1976): “A History of Metallurgy”. The Metals Society. Londres.

- (1987): "The Early History of Metallurgy in Europe". Longman. Londres.
TYLECOTE, R. y MERKEL, J. (1985): "Experimental smelting techniques: achievements and future". En P. Craddock y M. Hughes (eds.): Furnaces and Smelting Technology in Antiquity. British Museum. Londres: 3-20.

Voss, O. (1988): "The iron production in Populonia". En G. Sperl (ed.): The First Iron in the Mediterranean. Proceedings of the Populonia/Piombino 1983 Symposium. PACT, 21, Strasbourg: 91-100.

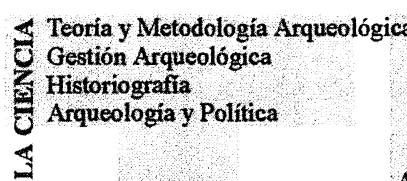

$$
{ }_{L^{A}} \text { CULTU }_{R_{A}}
$$

Arqueología Prehistórica y Protohistórica Paleolítico

Neolitico

Calcolitico

Edad del Bronee

Edad del Hierro

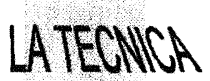

Datación absoluta

Arqueología analítica

Arqueometalurgia

Tecnologia experimental

Trabajos de Prehistoria es la revista de consulta imprescindible para todos aquellos interesados en conocer el estado de la cuestión sobre el rico-patrímonio arqueológico de la Prehistoria y Protohistoria de la Península Ibérica. Sus páginas reflejan tendencias punteras de su especialidad, por

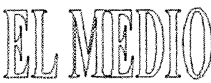

Arqueobotánica Arqueozoología Arqueología del Paisaje lo que figura en los más significativos repertorios bibliográficos nacionales e internacionales.

BOLETIN DE PEDIDO

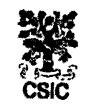

Servicio de Publicaciones

Teléfono: 91 / 5612833 Fax: 91 / 5629634 Vitruvio, 8 - 28006 Madrid (Espania)
Adjunto cheque bancario por valor de a nombre de Servicio de Publicaciones CSIC

Contra reembolso

Envienme, por favor, factura pro-forma

Tarjeta Visa No $^{\circ}$

Fecha de caducidad
TRABAJOS DE PREHISTORIA (Revista Semestral)

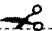

Nombre

Dirección Ciudad

\section{Ptas Suscripción anual \\ N. sueltos}

Fecha

Firma
Suscripción España: 5200 Ptas. Suscripción Extranjero: 7800 Ptas. $N^{\circ}$ suelto España: 3100 Ptas. $N^{\circ}$ suelto Extranjero: 4700 Ptas.

T. P., 53, n. ${ }^{\circ} 2,1996$ 